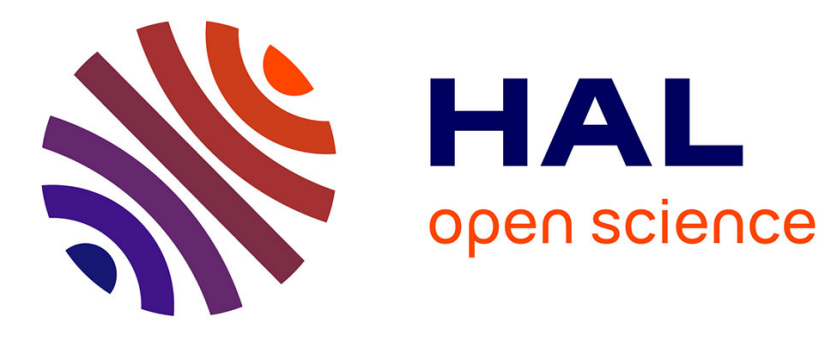

\title{
ACTAR TPC performance with GET electronics
}

J. Giovinazzo, J. Pancin, J. Pibernat, T. Roger

\section{To cite this version:}

J. Giovinazzo, J. Pancin, J. Pibernat, T. Roger. ACTAR TPC performance with GET electronics. Nucl.Instrum.Meth.A, 2020, 953, pp.163184. 10.1016/j.nima.2019.163184 . hal-02416890

\section{HAL Id: hal-02416890 https://hal.science/hal-02416890}

Submitted on 9 Nov 2020

HAL is a multi-disciplinary open access archive for the deposit and dissemination of scientific research documents, whether they are published or not. The documents may come from teaching and research institutions in France or abroad, or from public or private research centers.
L'archive ouverte pluridisciplinaire HAL, est destinée au dépôt et à la diffusion de documents scientifiques de niveau recherche, publiés ou non, émanant des établissements d'enseignement et de recherche français ou étrangers, des laboratoires publics ou privés. 


\section{ACTAR TPC performance with GET electronics}

J. Giovinazzo ${ }^{1 \mathrm{a}}$, J. Pancin ${ }^{\mathrm{b}}$, J. Pibernat ${ }^{\mathrm{a}}$, T. Roger ${ }^{\mathrm{b}}$

${ }^{a}$ Centre d'Etudes Nucléaires de Bordeaux Gradignan (CENBG) - UMR 5797, CNRS/IN2P3 - Université de Bordeaux, Chemin du Solarium, F-33175 Gradignan, France ${ }^{b}$ Grand Accĺ́ateur National dIons Lourds (GANIL), CEA/DRF - CNRS/IN2P3, Boulevard Henri Becquerel, 14076 CAEN, France

\footnotetext{
Abstract

The ACTAR TPC detector has been designed as a state-of-art detector for the tracking of ions in nuclear physics experiments. We performed various sets of test measurements with an alpha source, using a reduced size demonstrator of this device, in order to estimate the performances that can be expected from the tracks analysis. The parameters from the detector, from the electronics and from the analysis have been studied, with their influence on the energy resolution and the tracking precision. An effective full 3D charge distribution reconstruction method is also presented in order to analyze particle trajectories in any direction.

Keywords: ACTAR TPC, GET electronics, Track reconstruction, Detector performance

19 PACS: 07.05.Hd, 07.05.Kf, 29.40.Cs, 29.40.Gx
}

\section{Introduction}

The ACTAR TPC detector $[1,2,3]$ has been developped to address specific challenges of nuclear physics experiments aiming the observation of rare processes, reactions or decays, involving exotic nuclei. The purpose of such a detector is to perform an efficient tracking of the ions. In addition, a good measurement of the energy deposit of the particles along the tracks is also required for their identification.

The detector is based on the principle of a time projection chamber (TPC): the reaction or the decay of interest takes place in a gas volume, and the device measures the ionization signal along the particles tracks. ACTAR TPC uses a 2D $(X, Y)$ pixel pad-plane for the collection of the signal projected by mean of a uniform electric field. The third dimension $(Z)$ is analyzed with the time sampling of the signal on each pad. The readout of the collected signal of pads is done with the GET electronics [4] that has been developed with this purpose.

\footnotetext{
${ }^{1}$ Corresponding author: J. Giovinazzo, giovinaz@cenbg.in2p3.fr
} 
Several other detectors have been developed around the world, based on the same principle, and using the same electronics, such as AT-TPC at MSU [5, 6] and $\mathrm{S} \pi \mathrm{RIT}$ at RIKEN [7].

The ACTAR TPC device is a relatively complex instrument, that can be configured in many aspects: the detector conditions (gas type and pressure, drift electric field), the electronics settings, and the data analysis procedures. The purpose of present paper is to explore these settings in order to evaluate their impact on the quality of the measurements. The results presented in this work have been obtained with an alpha source, which is an interesting compromise between protons observed in exotic decay modes and light ions observed in nuclear reactions.

In the first section, we describe the set-up for the measurements and we present the most relevant parameters of the setting. The next section describes the various corrections and calibration processes to be applied to the raw signal of each pad, before the global analysis of the full events. Then the tracks are reconstructed, and using the analysis of the energy deposited, the performances are estimated in terms of energy resolution and track reconstruction precision. The fourth section shows the influence of the various settings on the performances of the device.

The measurements presented in the sections mentioned above are obtained with alpha particle trajectories that are relatively parallel to the pad-plane. This is a more comfortable situation in terms of tracks analysis since the 2dimension projection of the signal contains all the information. This is sufficient to demonstrate the effect of parameters on the results quality. Nevertheless, the analysis of vertical tracks require some more developed processing. In the last section, we propose a full 3D charge distribution reconstruction method that can be applied for the analysis of tracks in any direction.

\section{ACTAR TPC demonstrator test set-up}

The detector used for present work is the ACTAR TPC demonstrator, which is a reduced size (2048 pads) version of the final device. A full description can be found in ref. [2].

The active surface of the pad-plane is $X \times Y=12.8 \times 6.4 \mathrm{~cm}^{2}(64 \times 32$ pads of $2 \times 2 \mathrm{~mm}^{2}$ ) and the height of the gas volume is $17 \mathrm{~cm}$. The pad-plane is equipped with a bulk micromegas [8] for signal amplification. The signal generated on the mesh of the micromegas is used to define the trigger for the GET electronics (external trigger).

For all measurements analyzed in this article, we used a 3-alpha source $\left({ }^{239} \mathrm{Pu},{ }^{241} \mathrm{Am}\right.$ and $\left.{ }^{244} \mathrm{Cm}\right)$. The performance analyses of section 4 are done with the source located on the short side of the drift cage (see figure 1-left). For the development proposed in section 5 , the source was located on top of the active volume (figure 1-right).

Since the source is roughly collimated, the particles are emitted in a cone around the $X$ or $Z$-axis for a source on the side or on the top of the detector 
respectively, with a maximum opening angle about $\pm 40^{\circ}$. For the analysis, we consider only alpha particles that are stopped in the active volume: we reject events if the projected signal reaches the sides of the pad-plane. In addition, to limit signal collection distortions due to side effects of the field cage, the pads located along the border of the collection plane are not considered in the analysis.

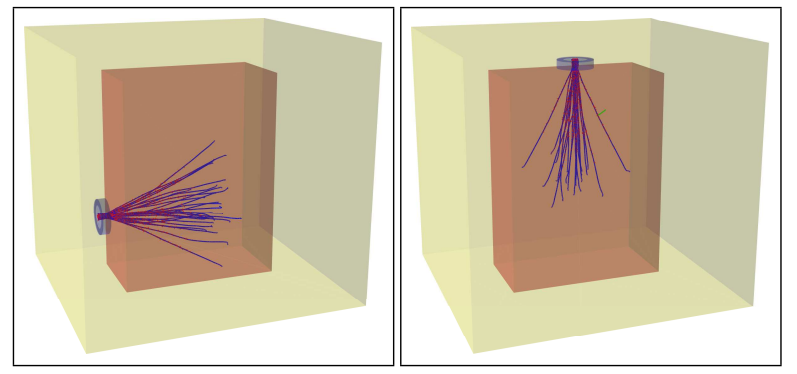

Figure 1: Position of the alpha source relative to the active volume (represented in dark color): on the side (left picture) resulting in tracks around the $X$ horizontal axis, or on the top (right picture) with tracks around the $Z$ vertical axis. In both cases the source is located in the gas chamber, but outside the active volume of the TPC. The pictures are from a Geant4 [9] simulation.

Since the source is located outside the drift cage (about $17 \mathrm{~mm}$ ), the alpha particles lose some energy before entering this volume. This dead zone needs to be taken into account in the analysis of the energy deposited by the particles in the detector (see section 3.3).

In the following paragraphs, we define the role of the various parameters and settings that are analyzed in the next sections of the paper.

\subsection{Detector settings}

The measurements are performed using P10 gas (90\% Argon, 10\% Methane). The gas pressure is set to 400 mbar in order to stop the particles with trajectories parallel to the source axis in the active volume. In this work, the gas pressure is not considered as a parameter to explore. Indeed, it is generally imposed by the purpose of the experiment: for example to stop protons in exotic decay studies, or to set the target thickness (and corresponding beam energy) when the detector is used as an active target. The influence of the voltage applied on the micromegas mesh (signal amplification) is also not considered here. It is set to $-340 \mathrm{~V}$ to adjust signal amplitude to the electronics dynamic range. This point is discussed in the next section.

The only effective parameter related to the detector settings is the high voltage applied on the TPC cathode that defines the electric field (in $\mathrm{V} / \mathrm{cm}$ ) responsible for the drift of ionization signals towards the pad-plane. This drift field affects mainly 2 quantities related to the tracks signal: 
- the drift velocity $v_{\text {drift }}$ of the ionization electrons, that is needed to convert the drift time into the $3^{\text {rd }}$ dimension Z: $z=v_{\text {drift }} \times t$;

- the dispersion of the signal in $\mathrm{X}$ and $\mathrm{Y}$ directions which corresponds to the transverse diffusion of the electrons while they drift along the Z-axis.

These quantities can be estimated for a given gas (here P10) at defined pressure and drift electric field with a code like GARFIELD [10]. We use values from GARFIELD for a first analysis, and further correct them with an empirical method (see section 3.2).

\subsection{Electronics parameters}

The pad-plane is connected to the GET electronics for the readout of the collected signal. For each channel, the main components of interest are the charge sensitive preamplifier (CSA), the shaper and the analog memory. For more details, see ref. [4].

In our study, the CSA only defines the dynamic range of the input signal, set by the input capacitors $C_{g}$. For all measurements presented here, it was set to $1 p C$ so that, according to the amplification from the micromegas, the maximum signal amplitude corresponds to an important fraction of the dynamic range and does not saturate the channels electronics.

The shaper stage is a filter with a peaking time $\tau$ that can be selected from 16 values between $70 \mathrm{~ns}$ and $1 \mu \mathrm{s}$. While the CSA converts the charge deposit on pads into a signal amplitude, the shaper/filter improves the signal over noise ratio for a better measurement of this amplitude, but also introduces a distortion that washes out the time structure of the input charge. While a larger peaking time provides a better filtering, a shorter one allows a better separation of multiple tracks along the $\mathrm{Z} /$ time axis (simultaneous particles in a vertical plane or pile-up event).

The output signal of the shaper is sampled in time and stored into the analog memory, to be further digitized by an ADC. The memory contains 512 capacitors (memory cells) that can store the signal with a write frequency $F_{W}$ ranging from 1 to $100 \mathrm{MHz}$. The GET electronics allows to read and process either all or only a reduced number of memory cells (the readout depth $N$ ), thus reducing the amount of data and the processing time.

For the analysis of the influence of the settings mentionned above, the measurements are performed using full readout mode of the GET electronics, where all channels are read and stored. Due to the amount of data generated, most experiments are foreseen to run in partial readout mode, reading only the channels with an effective signal. Each channel has a leading edge discriminator with a tunable threshold to define whether it is read or not in this mode.

\section{Raw data processing}

A single event corresponds to a partial or full set of pad signals registered as time samples with a number of data (time buckets) corresponding to the 
readout depth. The size of the time window of the sampled data is $N / F_{W}$ : it must be larger than the drift time through the full height of the active volume ( $Z$ axis).

Once the raw data from each pad are stored, some preliminary data corrections are required for the global analysis of the tracks. The first processing of raw data has been described in a previous paper [11] to correct for systematic effects from the measured signal, at the level of a single channel (section 2.1). This section also introduces additional corrections related to the detection system: the gain matching of pad signals (section 2.2) and the correction of the signal induced on all pads because of the whole charge collected on the micromegas mesh (section 2.3).

\subsection{Raw signal corrections}

The GET electronics are equipped with specific channels (fix pattern noise, or FPN) that can be used to suppress part of the coherent noise of the electronics. This is achieved on an event-by-event basis, and requires the storage of these FPN channels, which may represent the same amount of data as the signal channels for an experiment in partial readout mode. An alternative method is also presented in ref. [11].

This paper also suggests a fixed baseline (BL) correction for each channel since the average samples registered in absence of input signal do not correspond to a constant value. A specific baseline is estimated for each channel, with or without the previous FPN correction.

Two additional corrections have been considered in this work for individual pad signals. The first correction is an automatic offset to correct for slow (low frequency) baseline variations. The trigger delay, that tells the GET electronics to stop writting data in the analog memories, is set so that the beginning of the registered channel data samples is well before the effective signal (1 to $2 \mu \mathrm{s}$ ). Then the first part of the sample is averaged to estimate the constant offset to be applied to force the baseline to 0 . The second correction to channel signals is the smoothing of the time sampled data (with a Gauss function) to reduce the noise. When applying this correction, the width of the convolution is set to the order of the sampling period, typically $20 \mathrm{~ns}$ for a $50 \mathrm{MHz}$ write frequency.

\subsection{Pads calibration}

In order to sum the signal amplitude of the pads (for total energy deposited) or to compare them (for signal distribution in track analysis), it is necessary to calibrate each channel with respect to a common reference. Rather than a real calibration, we perform a gain matching of the each channel's amplification, as described in ref. [2].

This procedure is based on the measurements of the signal on all pads, induced from pulses of different amplitudes applied to the micromegas mesh. From such a set of data, the gain matching needs to be computed according to the other corrections applied (FPN and baseline corrections, see 2.1) and to the GET electronics parameters (dynamic range and peaking time, but it does not depend on the write frequency). 
A slighly more precise calibration can be obtained with a full scanning of the pad plane as proposed in ref. [12], but such a scan is not available for the present work.

\subsection{Mesh-induced signal correction}

When the ionization signal is amplified by the micromegas, this creates a global current on the mesh that generates an induced signal on all pads. This signal has an opposite polarity compared to the ionization collection. The resulting negative component, that distorts the signal measured for all pads, can be estimated and corrected as illustrated in figure 2, from the data measured for pads located outside the track. This component is then subtracted from all signal channels.

In order to apply this correction in partial readout mode, since only pads with signal are read, it is necessary to force the readout of a set of pads randomly distributed on the pad plane to ensure that most of these pads have a high probability to be outside the events tracks.

As shown in figure 2(d), this correction modifies the amplitude of the signal, but also the timing information when considering signal time as the position of signal maximum or when estimating this time by a CFD algorithm.

\subsection{Summary of correction}

Figure 3 summarizes the effect of the data corrections presented in this section on the energy resolution estimated from the 3 components of the alpha source (see section 3.3). It compares the cases where the FPN correction is applied or not.

All results from this figure have been obtained from the same events. This measurement, used as a reference in the next sections, is performed with the following parameters:

- $H V_{\text {drift }}=75 \mathrm{~V} / \mathrm{cm}$ drift high voltage;

- $C_{g}=1 p C$ input dynamic range, $\tau=502 n s$ peaking time, $N=256$ readout depth and $F_{W}=25 \mathrm{MHz}$ write frequency for the GET electronics.

The sampling time window $\left(T_{\text {mes }}=N / F_{W}=10.24 \mu s\right)$ is large enough to include the baseline estimate window, the full drift time $(\sim 4 \mu s$ for a drift height of $170 \mathrm{~mm}$ and a drift velocity estimated to $40 \mathrm{~mm} / \mu \mathrm{s}$ ) and few times the peaking time (signal width).

Except for the smoothing of the samples, all other corrections have a measurable effect on the estimated energy resolution. As a consequence, we recommend the following corrections to be considered for the ACTAR TPC data analysis:

- FPN correction (coherent noise reduction);

- channels baseline (FPN corrected) subtraction;

- channels gain matching (here, from pulser signal on mesh); 

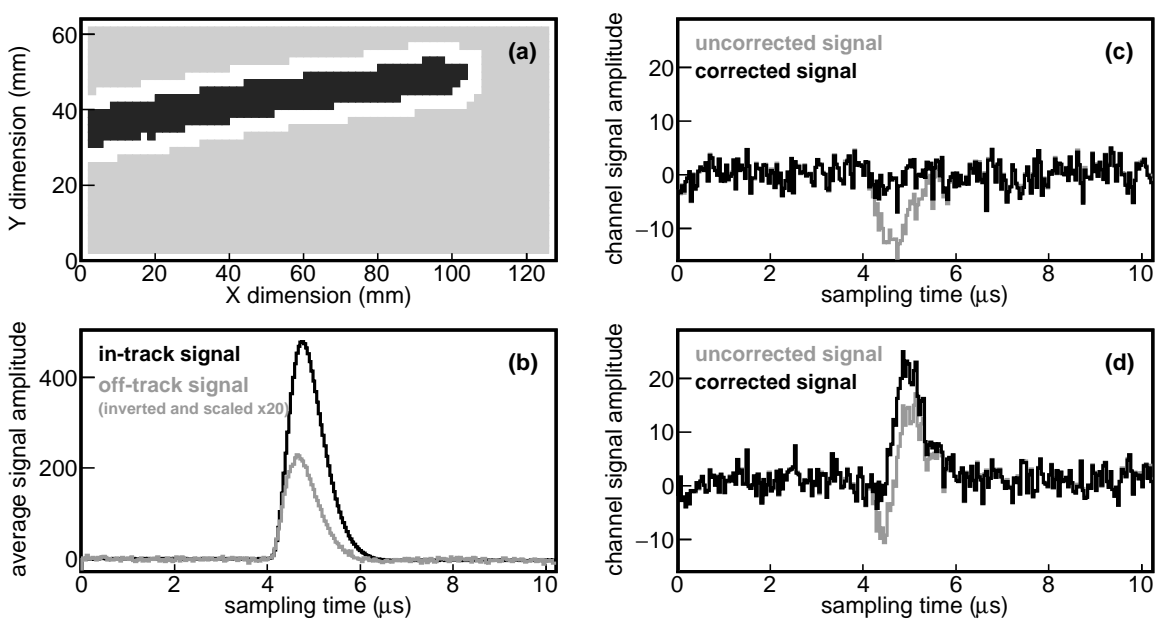

Figure 2: Illustration of the correction of the signal induced on all pads by the micromegas mesh. Figure (a) shows in black the pads with signal from a particle track, while the off-track pads in gray are used to estimate the induced signal. The pads located at the border between in- and off-track are excluded from this correction, because they may contain a signal component that is too small to define if the pad is in or out of the track. The average signal of the off-track pads is plotted in gray in figure (b), inverted and scaled for comparison with the average signal of in-track pads in black. Figures (c) and (d) show the uncorrected (in gray) and corrected (in black) signals for a pad outside the track and on the side of the track respectively. The amplitudes in plots (a), (b) and (c) are given in coder units.

- automatic offset correction;

- induced signal correction from off-track pad signals.

\section{Analysis procedure}

In this section, we describe the events analysis with the data corrected as described in the previous section. It is performed here on the $2 \mathrm{D}$ amplitude and timing information from the pad signals: for a pad $\left(i_{x}, i_{y}\right)$, the signal amplitude $A\left(i_{x}, i_{y}\right)$ is the maximum of the signal distribution measured in the pad electronic channel, and the associated time $T\left(i_{x}, i_{y}\right)$ using a digital constant fraction discriminator algorithm (as proposed in ref. $[2,11]$ ). In order to select the pads with an effective signal, an analysis (software) threshold is defined. This threshold is expressed in coder units (about $1 \mathrm{~V}$ for 12 bits digitization), because the data corrected according to section 2 are time sampled signals expressed in this unit (as in figure 2). 


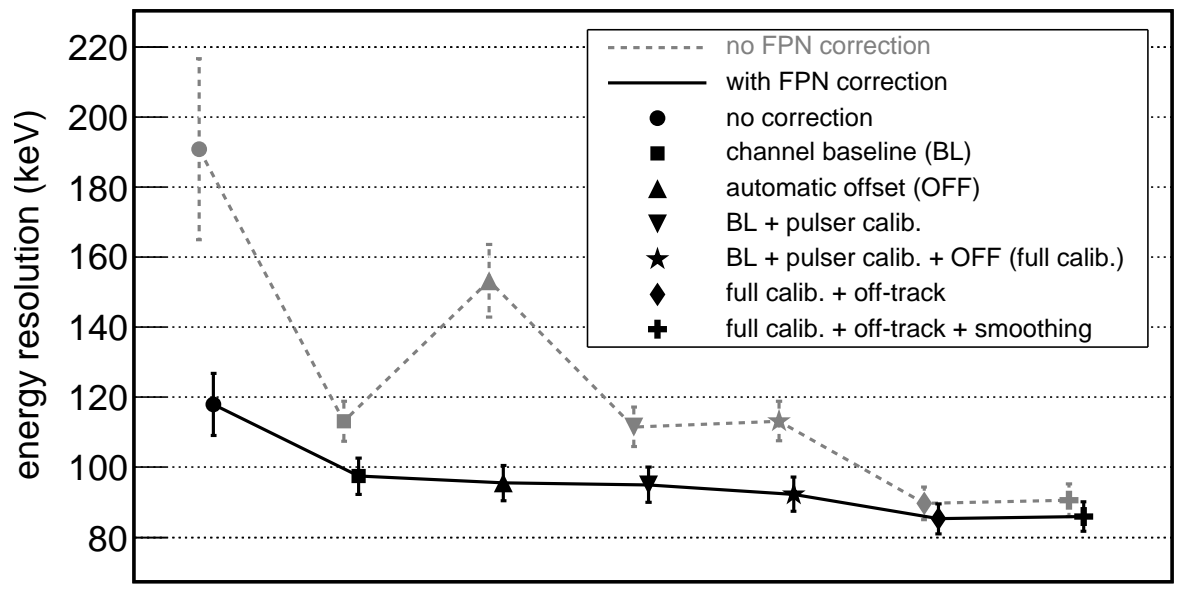

Figure 3: Effect of the data corrections on the energy resolution (FWHM) estimated from the energy fit of the 3 peaks of the alpha source. The points connected with a plain line (red online) correspond to the energy resolution when first applying the FPN correction, the points connected with a dashed line (blue online) when this correction is not applied. All the points result from the analysis of the same set of events, with a maximum emission angle of $15^{\circ}$ (see section 3.3).

This $2 \mathrm{D}$ analysis is valid for the performances estimate since the alpha particles are emitted with limited angles with respect to the horizontal pad-plane. Some limitations of this analysis are mentioned in section 5 . The results shown in this section are obtained with the same reference measurement as in 2.4.

\subsection{Tracks fitting}

For the analysis of the alpha particles tracks, the pad signals amplitude and time are fitted as proposed in ref. [2].

A geometrical curve parametrization defines the path of the particle track in the 3D active volume $P_{t r}(\varepsilon)=\left(x_{t r}(\varepsilon), y_{t r}(\varepsilon), z_{t r}(\varepsilon)\right)$, with a curve coordinate $\varepsilon: P_{t r}(\varepsilon=0)$ is the particle starting point and $P_{t r}(\varepsilon=1)$ is the stopping point. The curve coordinate is defined so that, for each track, the length $L(\varepsilon)$ uniformly varies with $\varepsilon$ :

$$
L(\varepsilon)=\varepsilon \cdot L_{\text {track }} \text { with } \varepsilon \in[0 ; 1]
$$

where $L_{\text {track }}$ is the length of the track.

The model uses a scalable Bragg peak pattern for energy loss along the particle path $f_{E}(\varepsilon \mid A, \lambda$ ) (with an amplitude parameter $A$ and a length parameter $\lambda$, see ref. [2] section 4.4.3 and fig. 12). 
The 2D signal amplitude function is then:

$$
A_{X Y}(x, y)=\int_{\varepsilon=0}^{1} F_{X Y}(x, y, \varepsilon) \cdot d \varepsilon
$$

258 with $F_{X Y}(x, y, \varepsilon)$ being the contribution of the energy deposited at $\vec{P}_{t r}(\varepsilon)$ to ${ }_{259}$ the charge collected at projection position $(x, y)$ :

$$
F_{X Y}(x, y, \varepsilon)=f_{E}(\varepsilon) \cdot D_{X Y}(x, y, \varepsilon)
$$

where $f_{E}(\varepsilon)$ is the Bragg peak parametrization and $D_{X Y}$ is the signal dispersion on pad plane. The dispersion is considered Gaussian in $X$ and $Y$ dimensions, with the same width $\sigma_{X Y}$ and taking into account a $Z$ dependence, $\sigma_{X Y}(\varepsilon)=$ $\sigma_{X Y}^{0}+\sigma_{X Y}^{1} \cdot \sqrt{z_{t r}(\varepsilon)}$ :

$$
D_{X Y}(x, y, \varepsilon)=\frac{1}{2 \pi \cdot \sigma_{X Y}(\varepsilon)^{2}} \cdot e^{-\left[\frac{\left(x-x_{t r}(\varepsilon)\right)^{2}}{2 \cdot \sigma_{X Y}(\varepsilon)^{2}}+\frac{\left(y-y_{t r}(\varepsilon)\right)^{2}}{2 \cdot \sigma_{X Y}(\varepsilon)^{2}}\right]}
$$

The associated 2D timing information is build from the weighted average of contributions from track points (with $t_{t r}=z_{t r} / v_{d r i f t}$ ):

$$
T_{X Y}(x, y)=\frac{\int_{\varepsilon=0}^{1} t_{t r}(\varepsilon) \cdot F_{X Y}(x, y, \varepsilon) \cdot d \varepsilon}{\int_{\varepsilon=0}^{1} F_{X Y}(x, y, \varepsilon) \cdot d \varepsilon}
$$

An illustration of the pad signals amplitude fit is shown in figure 4 . The simplest case for the trajectory model is to consider a segment from the start point $P_{0}$ to the stop point $P_{1}$ of the particles (in this case, $\vec{P}_{t r}(\varepsilon)=(1-\varepsilon) \cdot \vec{P}_{0}+$ $\left.\varepsilon \cdot \vec{P}_{1}\right)$. Since we observe events with small deviations from a straight trajectory, other models have been tested, such as a 3-points Bézier curve defined with 3 points, or a multiple segments path. These models allow for a better fit of the measured tracks, due to the scattering of the alpha particle in the gas.

In the present work, we focused on a 2 -segments (3 points) model that give better results than the linear or Bézier tracks. As a comparison, figure 5-left shows the distributions of the alpha particle emission point coordinate $y_{t r}(\varepsilon=0)$ resulting from the fit with a linear or a 3-point segments track curves (respective half-width of 5.2 and $3.2 \mathrm{~mm}$ ). This illustrates the better quality of the segments trajectory model (the effective source $Y$ extension is limited by a $5 \mathrm{~mm}$ collimator, as in figure 1-left).

The comparison of the fitted track length resolution is shown in figure 5right, and the resolution is also slightly better with the 2-segments model.

These models for trajectory are effective as long as the particle is not subject to strong scattering effects. In few cases (less than $0.5 \%$ of the events), the trajectories show a large deviation angle, that is attributed to Rutherford backscattering. In such cases, the fitting model is not well adapted because of the energy transfer at the scattering point. 

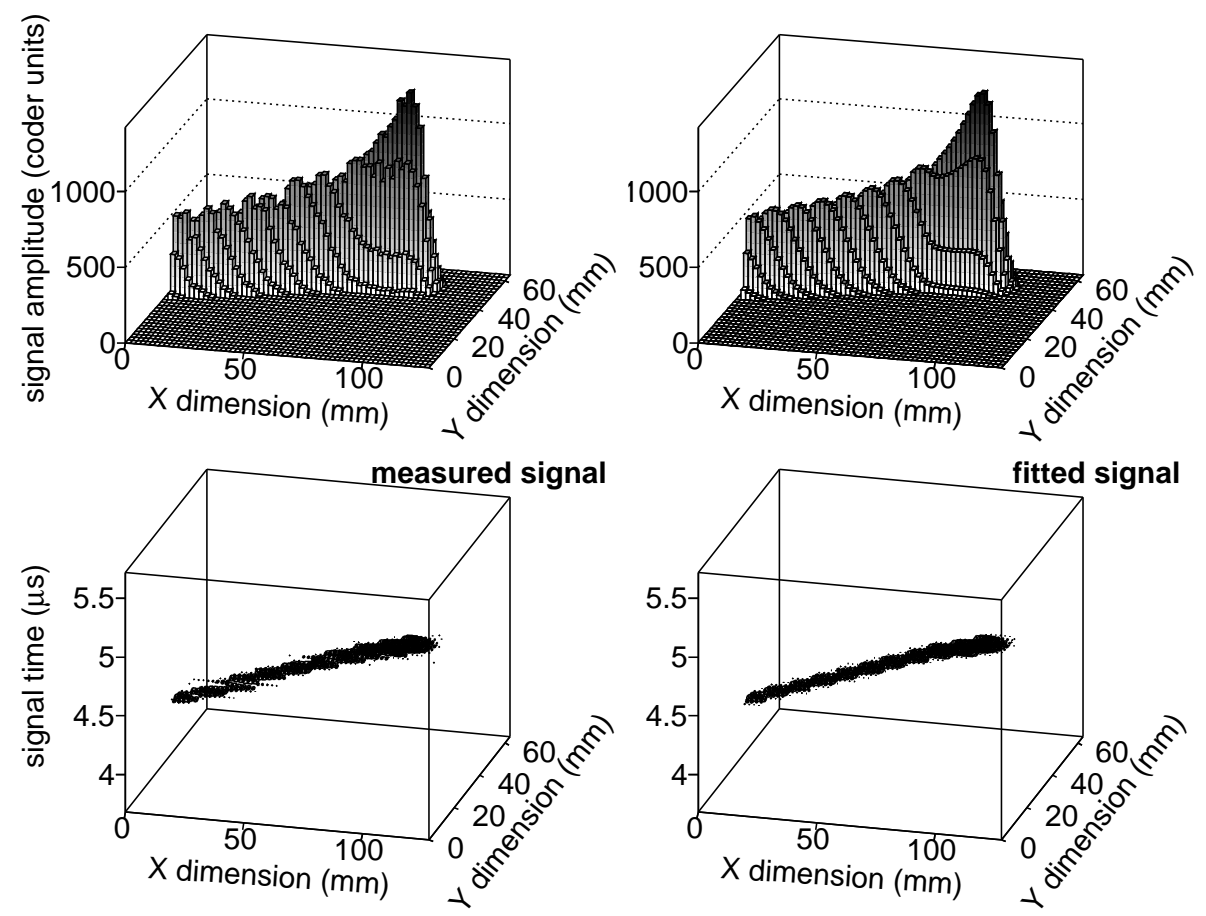

Figure 4: Illustration of the tracks fitting: comparison of the measured data (left plots) with the fit results (right plots). The upper plots show the pad signals amplitude $A\left(i_{x}, i_{y}\right)$. The lower plots represent the pad signals with $3^{\text {rd }}$ dimension corresponding to the time information: from CFD analysis for measured signal (left) and from eq. 5 for the fit (right). The event has been fitted with a 3-points segments trajectory (see text).

The fit is performed simultaneously on signal amplitude $A(x, y)$ (eq. 2) and timing $T(x, y)$ (eq. 5$)$. The fit parameters are:

- the track curve definition points in $(x, y, z) 3 \mathrm{D}$ space: 6 parameters $\left(P_{0}=\right.$ $\left(x_{0}, y_{0}, z_{0}\right)$ and $\left.P_{1}=\left(x_{1}, y_{1}, z_{1}\right)\right)$ for a single segment track, 9 parameters for curves defined with 3 points such as the 2-segments curve used in this paper (the source, when located on the side of the active volume is at position $x_{0} \simeq-17 \mathrm{~mm}$ and the corresponding parameter is fixed);

- 2 parameters for the scalable Bragg peak model $(A$ and $\lambda)$;

- 2 parameters for the signal dispersion with $Z$ dependence $\left(\sigma_{X Y}^{0}\right.$ and $\left.\sigma_{X Y}^{1}\right)$; 

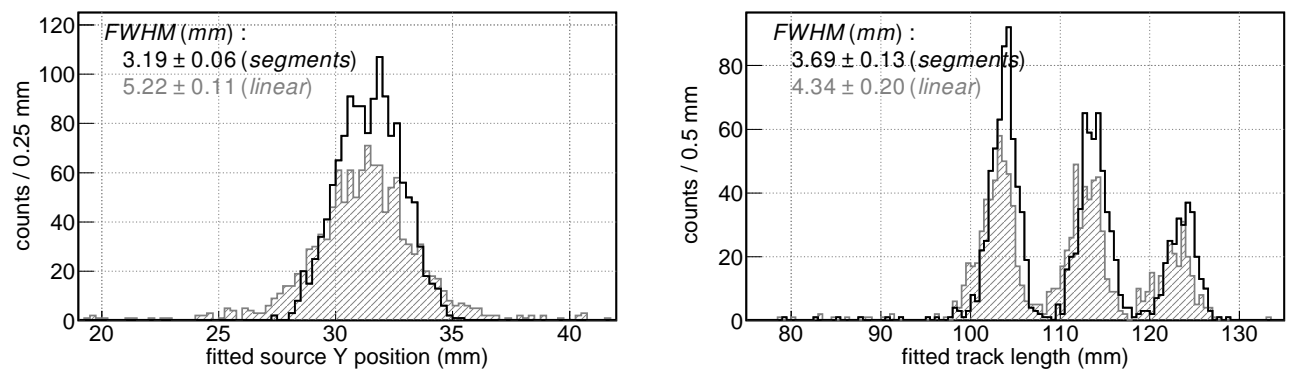

Figure 5: The left plot shows the comparison of the source $Y$ position distribution resulting from tracks fit with a linear (single segment) trajectory (hatched gray plot) or with a 2-segments (plain black line) one. The right plot shows the alpha particles trajectory length from the events fitted the same models (see section 3.2). The displayed values correspond to the width of the center peak.

\subsection{Track length and drift velocity}

The fit of tracks is performed in the $(X, Y)$ horizontal coordinates of the pad-plane and the sampling time coordinate $T$ for the $3^{\text {rd }}$ dimension (vertical). To extract the effective track length from the curve definition parameters of the fit, the time coordinates $t$ need to be translated into the space coordinate $z$, by mean of the drift velocity $v_{\text {drift }}: z=v_{\text {drift }} \cdot t$.

An empirical method is proposed in ref. [2] to estimate the drift velocity by minimizing the fitted length resolution. The drift velocities obtained with this method are used for the data analyzed in present work.

The length distribution resulting from the fit of the 3 -alpha source tracks (see fig. 5-right) is fitted with 3 independant Gaussian terms. For the comparison of the performances of the device when changing setting parameters, we use the center peak width. The mean track length for each peak (centroid) is not expected to vary with setting parameters under study since it depends only on the gas pressure. For the performance analysis (section 4) relative to track fitted-length resolution, one should keep in mind that a Geant4 simulation [9] provides an intrinsic FWHM of about $3.2 \mathrm{~mm}$ [2] due to the slowing processes and interactions (independently of the measurement quality).

\subsection{Energy resolution}

The energy deposited in the gas volume is estimated by summing the signal amplitude of all hit pads. As in ref. [2], the energy resolution can be estimated with a fit of the peaks corresponding to average energy of each of the 3 alphaemitting sources.

Because of the energy loss in the dead zone before the active volume, the collected signal corresponds to a reduced deposited energy. The energy lost in 


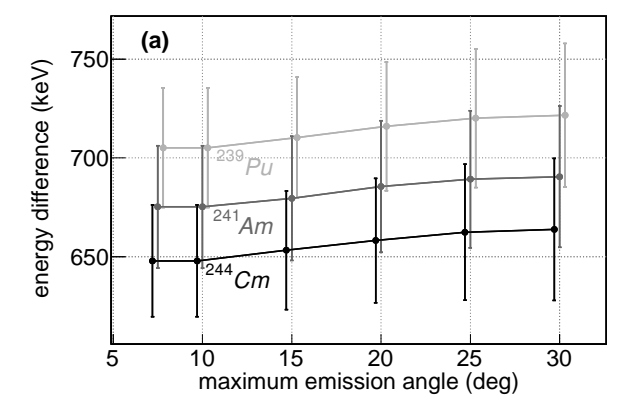
with the source emission opening angle (fig. 6). angles estimated by the track fits (see 3.1).

the dead zone is estimated with a Geant4 [9] simulation (including the collimator, that affects the angular distribution of detected events). Since the length travelled by the particles in the dead zone depends on the emission angle $\theta_{X}$ with respect to $\mathrm{X}$-axis, the average and the dispersion of the energy loss varies

This introduces a broadening of the measured energy peaks. As a consequence, in order to estimate the energy resolution (FWHM), we only select events with a limited angle (figure 7). This event selection is based on the

Figure 6: The left plot (a) is the average energy loss from the simulation for the 3 alpha emitters of the source, for various limitations of the emission angle. The error bars represent the width of the simulated peaks due to the energy loss dispersion. The right plot (b) shows the measured evolution of peaks position when restricting the analysis to events with a maximum angle. The error bars correspond to the estimated FWHM.

For the energy resolution estimates in the next section, the total signal amplitude distributions are fitted ( 3 peaks) and calibrated using the average peaks energies corrected for the energy loss in the dead zone. The events are selected with a maximum angle $\left(\theta_{X}\right)$ of $10^{\circ}$ to $15^{\circ}$, depending on the statistics of the measurements.

\section{Performances evolution with parameters}

The analysis procedure described previously is applied to measurements performed with various setup conditions or parameters. Each set of measurements corresponds to the variation of a selected parameter. Since the different sets have been performed at different moments over several months, the conditions between sets may vary slightly (such as temperature or atmospheric pressure that are not monitored), so we do not compare runs from different sets. 


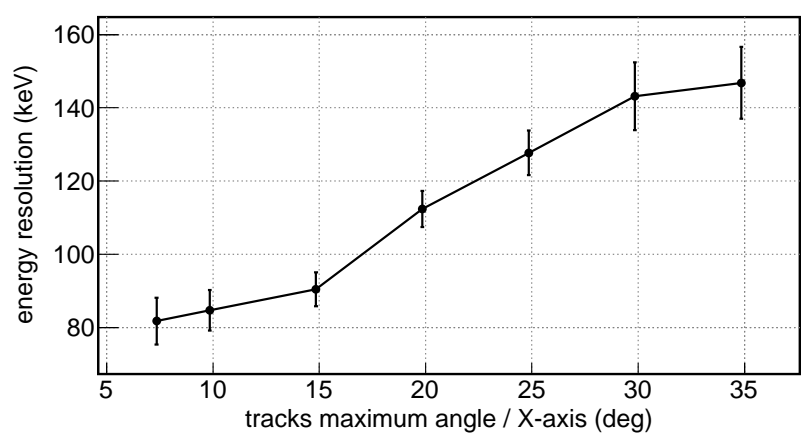

Figure 7: Estimated energy resolution variation with maximum incidence angle $\theta_{X}$ of alpha particles. The apparent stop of the increase of the measured resolution at $40^{\circ}$ is due to the collimator that stops the particles emitted at larger angles.

\subsection{Drift voltage}

The variation of the drift voltage is not expected to change significantly the precision of the results: it influences the drift velocity and the signal dispersion, but may not change the charge collection.

The measurements have been performed in full readout mode with $C_{g}=1 p C$ input dynamic range, $\tau=502 \mathrm{~ns}$ peaking time, $N=256$ readout depth and $F_{W}=25 \mathrm{MHz}$ write frequency for the GET electronics. The analysis threshold is set to 20 coder units.

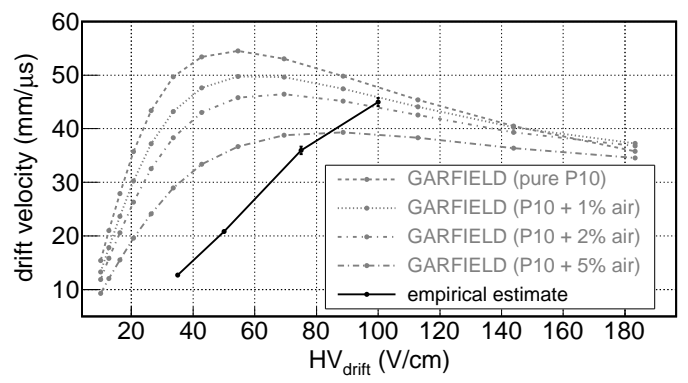

Figure 8: Drift velocity estimated (see section 3.2 and ref. [2]) for measurements with variation of the drift high voltage. The data (black points) are compared to the calculation of GARFIELD program [10] (gray points) for pure P10 gas or considering a 1\%, $2 \%$ and $5 \%$ air contamination (approximated as $80 \%$ nitrogen and $20 \%$ oxygen).

The drift velocity estimated for the measurement serie with drift high voltage variation is plotted in figure 8. The results are compared with the GARFIELD 
program [10] indicating an important disagreement of the drift velocity trend with high voltage, that is much beyond the uncertainties in gas pressure and temperature. As described below, there is a small leak in the detector chamber that could be responsible of a gas pollution with air in the percent level, but this does not explain the observed differences.

Figure 9 shows the energy and the fitted track length resolution evolution with drift high voltage, with an unexpected degradation of the resolution at low voltage.
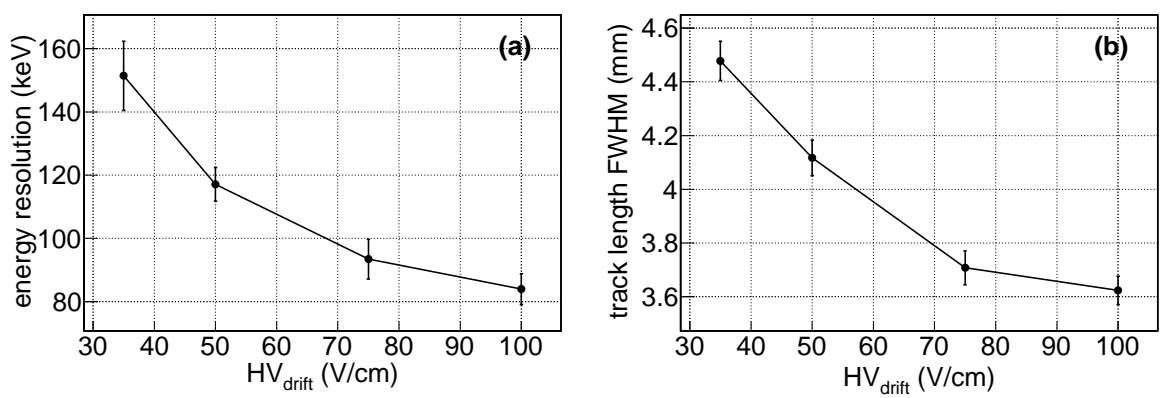

Figure 9: The left plot (a) shows the energy resolution and the right plot (b) shows the fitted length resolution, for various drift voltages. For energy resolution, only tracks with an emission angle $\theta_{X}<10^{\circ}$ have been considered.

For this set of measurements, figure 10 gives a clear illustration that there is a small gas contamination due to a leak in the detector chamber. A tiny fraction of air in the chamber induces the presence of oxygen that is responsible for a significant loss of drift electrons (attachment) along the drift path. For lower drift voltage (and lower drift velocity), there is a significant decrease of the collected signal (figure 10a). This indication of a gas contamination by oxygen is confirmed by figure 10b which shows that we observe a loss of the collected signal that increases with the drift length and that is larger for slower drift velocity.

Nevertheless, the signal loss due to gas contamination may not be the only cause of the worse resolution at low voltage. For the energy measurement, the FWHM is estimated for tracks with a small angle with respect to horizontal $X$-axis $\left(\theta_{X}<10^{\circ}\right)$. In that case, the drift length is almost constant along the tracks and the losses should be uniform. In addition, for $H V_{\text {drift }}=35 \mathrm{~V} / \mathrm{cm}$, the collected signal about $12 \%$ smaller than for $H V_{d r i f t}=100 \mathrm{~V} / \mathrm{cm}$ (figure 10a) and the resolution difference cannot be explained by the signal over noise reduction.

When needed, it is possible to apply a correction to the collected signal with a dependence on the $Z$ position along the particle trajectory, as in [12]. Since we limit the analysis to almost horizontal tracks in further analysis and to drift voltage larger than $75 \mathrm{~V} / \mathrm{cm}$, such a correction is not considered here.

Finally, despite a clear evidence of a small contamination, we conclude that 


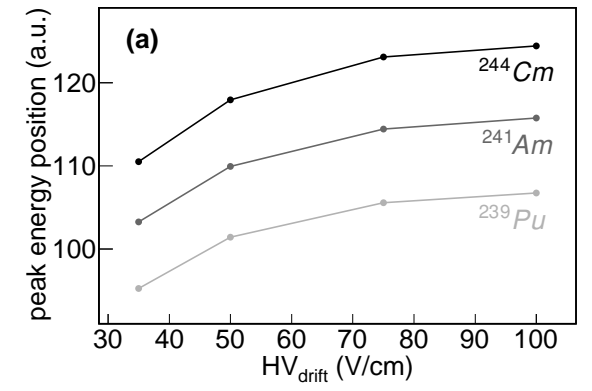
are used.

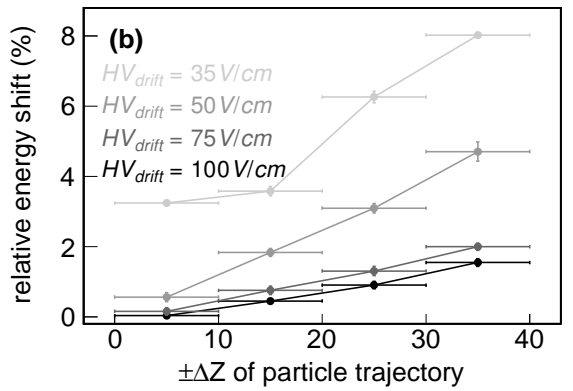

Figure 10: The left picture (a) shows the position of the energy peaks from the 3 components of the alpha source for different values of the drift voltage. On the right plot (b), we consider 4 intervals for $Z$ projection of the tracks $(\Delta Z=[0 ; 10]$, $[10 ; 20],[20 ; 30]$ or $[30 ; 40] \mathrm{mm}$ ) and for each interval, we consider the collected signal average (for the 3 alpha peaks) for tracks with $Z<0$ (towards the pad plane, $Q_{[-\Delta Z]}$ ) and for tracks with $Z>0$ (towards the drift cathode, $Q_{[+\Delta Z]}$ ) and we compute the relative difference. This difference is averaged for the 3 peaks of the source. For larger $Z$ intervals, the drift length difference between $Z<0$ and $Z>0$ tracks is larger, and the difference $Q_{[-\Delta Z]}-Q_{[+\Delta Z]}$ increases, showing the dependence of the signal loss with drift path length. This is plotted for different drift voltages, and the signal loss is more important (larger slope) for lower drift velocity.

the resolution (for both energy and track length) is better for increasing drift standard alpha source can be used to estimate it in the effective experimental gas conditions. For the tracks analysis of present paper, the empirical values

\subsection{Partial readout and channel trigger threshold}

In this section, we compare the full readout mode (all channels are read when an event is triggered) to the partial readout mode (only channels with signal above a defined threshold are read; for these channels, the full signal - all the time buckets - is read). The influence of the channels threshold is presented: we varied it from 4 to 48 . It is coded on 7 bits (128 values) covering $17 \%$ of the dynamic range $[13,4]$. This approximately corresponds to a variation from 22 to 260 coder units. In all cases, the analysis threshold (see section 3 ) was set to 15 coder units.

The measurements have been performed with the following common settings:

- $H V_{\text {drift }}=75 \mathrm{~V} / \mathrm{cm}$ drift high voltage; 
- $C_{g}=1 p C$ input dynamic range, $\tau=502 n s$ peaking time, $N=256$ readout depth and $F_{W}=25 \mathrm{MHz}$ write frequency for the GET electronics.

Figure 11a shows the evolution of the energy resolution with the channels threshold: for low values, it is comparable to the resolution obtained in full readout mode. A significant increase of the FWHM is observed above a threshold value of about 20. To a large extent, this can be explained by the fact that pads on the side of the track, with a small signal due to drift dispersion, are not read any more, resulting in the loss of part of the signal (as illustrated in figure 11b).
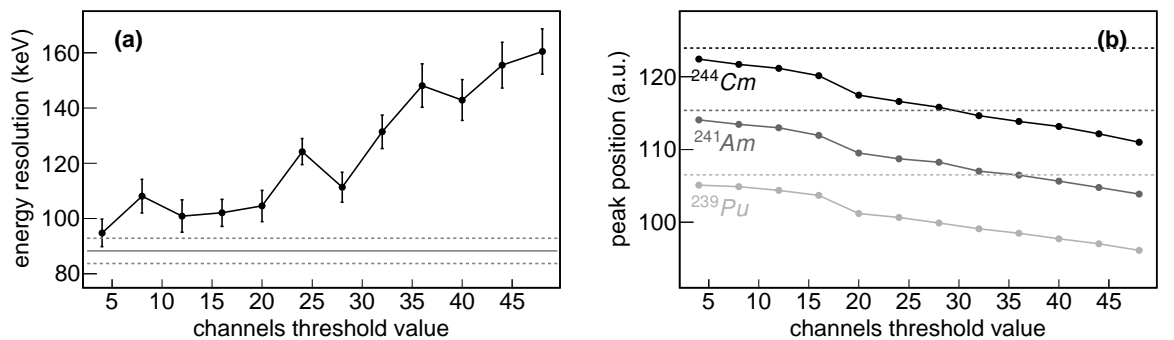

Figure 11: The left plot (a) shows the evolution of energy resolution with the channels threshold in partial readout mode (black points), and compared to the result in full readout mode (gray line). In this energy analysis, the tracks are selected with $\theta_{X}<15^{\circ}$. The right plot (b) illustrates the evolution of the signal for the 3 peaks of the alpha source.

The influence of the channels threshold value is less significant for the resolution of the fitted tracks length (figure 12). It is nevertheless not as good in partial readout mode as in full readout mode. This indicates that the pads on the side of the tracks have an influence on the fitting procedure.

\subsection{Readout depth}

The readout depth is the number of data that are processed to build the sampling of the signal for each channel (see 1.2). Since the sampling time window $T_{\text {mes }}$ is defined to include all required signal (see 2.4), it is kept constant when changing the readout depth. As a consequence, in order to compare results with different readout depths, the analog memory write frequency needs to be changed accordingly: $F_{W}=N / T_{\text {mes }}$.

We consider the following sampling parameters: $(N=512$, the full memory; $\left.F_{W}=50 \mathrm{MHz}\right),\left(N=256 ; F_{W}=25 \mathrm{MHz}\right)$ and $\left(N=128 ; F_{W}=12.5 \mathrm{MHz}\right)$. The common settings are:

- $H V_{d r i f t}=75 \mathrm{~V} / \mathrm{cm}$ drift high voltage; 


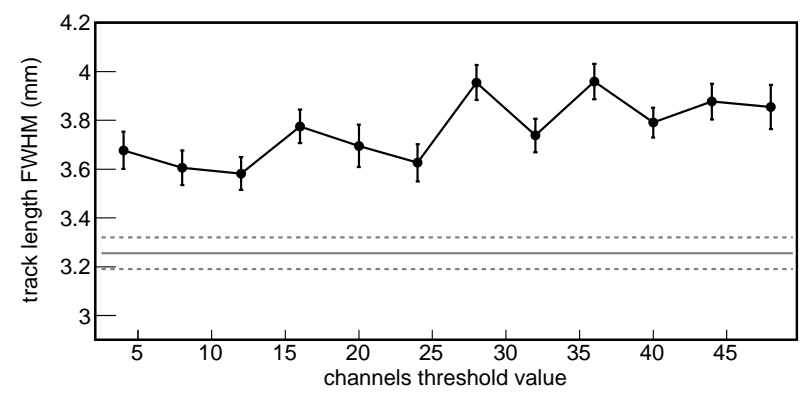

Figure 12: Variation of the length resolution of fitted tracks with the channels threshold (black), compared to the full readout mode (gray line).

- $C_{g}=1 p C$ input dynamic range, $\tau=502 \mathrm{~ns}$ peaking time in full and partial readout mode for the GET electronics (for partial readout, the channel threshold is set to 16 - see 4.2);

- analysis threshold set to 30 coder units.
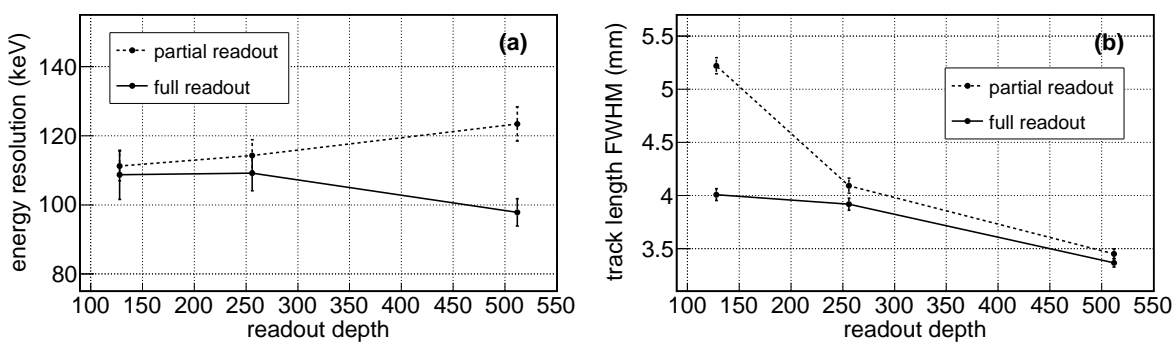

Figure 13: Energy (a) and fitted track length (b) resolution variation with readout depth. For energy resolution, the tracks are limited to $\theta_{X}<15^{\circ}$.

Figure 13 shows the energy and fitted track length resolutions for different readout depth settings, tested in both full and partial readout modes. From these results, we only conclude to a small decrease of the fitted length quality when reducing the sampling dimension. This should be considered carefully since the measurements were performed with a $\tau=502 \mathrm{~ns}$ peaking time, which is larger than the sampling periods considered (20, 40 and $80 \mathrm{~ns}$ ). Very short peaking time values should be more affected by the change of the write frequency.

For the settings used here, it is interesting that only a slight degradation is observed when changing from $N=512$ (with $F_{W}=50 \mathrm{MHz}$ ) to $N=256$ (with $F_{W}=25 \mathrm{MHz}$ ). This allows to run experiments with $N=256$ without 
any significant deterioration of the measurement quality, with the advantage of a factor 2 reduction of the acquisition dead-time and the volume of stored data.

\subsection{Shaper peaking time}

The last parameter we evaluated is the peaking time selected for the shaper of each channel. We performed measurements for $\tau=70,117,280,383$ and $502 \mathrm{~ns}$ nominal values of the GET electronics. The other settings are:

- $H V_{\text {drift }}=75 \mathrm{~V} / \mathrm{cm}$ drift high voltage;

- $C_{g}=1 p C$ input dynamic range, full readout, $N=512$ readout depth and $F_{W}=50 \mathrm{MHz}$ write frequency for the GET electronics;

- analysis threshold set to 30 coder units.

The comparison of results for the different peaking time values are reported in figure 14. It shows a clear trend of a better resolution for larger peaking time values.
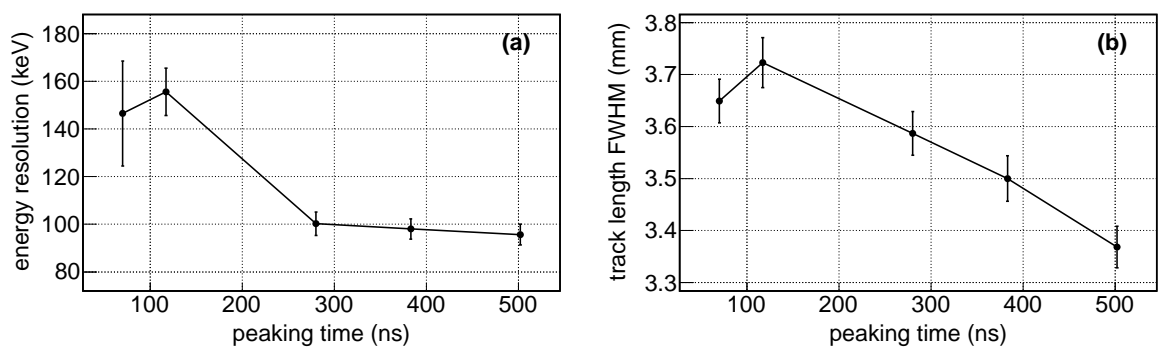

Figure 14: Energy (a) and fitted track length (b) resolution variation with peaking time. For energy resolution, the tracks are limited to $\theta_{X}<15^{\circ}$.

We suggest two reasons to explain this result. First, as shown in figure 1 of ref.[11], for the same input signal, the amplitude is approximately the same for all peaking times (so that the dynamic range for the ADC does not depend on this parameter), but then the integral signal increases with peaking time. As a consequence, a larger peaking time induces more filtering of the signal and a better signal over noise ratio. This explains the slight improvement observed in figure 14a for peaking times between 300 and $500 \mathrm{~ns}$. The second reason is due to the charge collection of the micromesh signal that is few hundred $n s$ long. For lower peaking times, this results in a ballistic deficit of the amplification stage and a loss in signal amplitude that is responsible for the resolution degradation at lower peaking times. 


\section{Reconstructed input signal analysis}

All the measurements used in the performance analysis of section 4 were realized with a roughly collimated source located on the side of the detector, providing tracks almost parallel to the collection plane. In that case, the ionisation signal above a single pad is created and collected in a short time, resulting in a short input charge that is processed by the charge preamplifier and the shaper of the channel. The maximum amplitude measured for the channel, which is equivalent to a peak sensing $\mathrm{ADC}$ value, is proportional to the collected charge.

This is not the case any more for close-to-vertical tracks. Such tracks may be measured for example in decay experiments, where an ion is stopped in the gas volume and decay particles can be emitted in any direction. In the extreme case of a perfectly vertical track, all the signal is created above a single pad (if we consider no dispersion) with a long time duration corresponding to the drift time difference between the upper and lower parts of the track. In that case, the amplitude of the shaped signal is no longer proportional to the total charge. Instead of the channel maximum amplitude, to measure the total energy deposited for a track one should use the signal integral (see figure 15a and 15b).

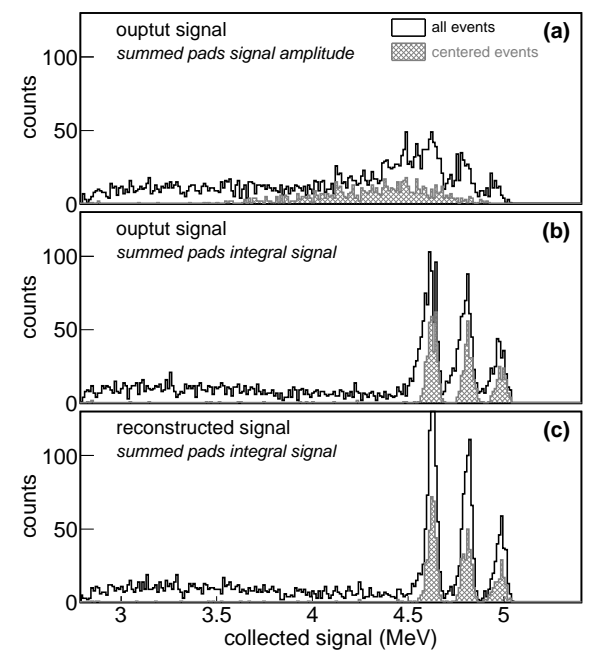

Figure 15: Energy distribution measured for alpha particles from the source located on top of the drift volume (see figure 1-right). The plots are: (a) the sum of the pad signal amplitudes from the channel output samples, (b) the integration of this output signal over the analysis threshold, and (c) the integration of the reconstructed input signals as proposed in section 5.1. For each plot, the black histogram is the result for all events, including alpha particles escaping the drift volume; the hatched gray histogram corresponds to a selection of centered events, with hit pads located in a limited $(X, Y)$ range. 


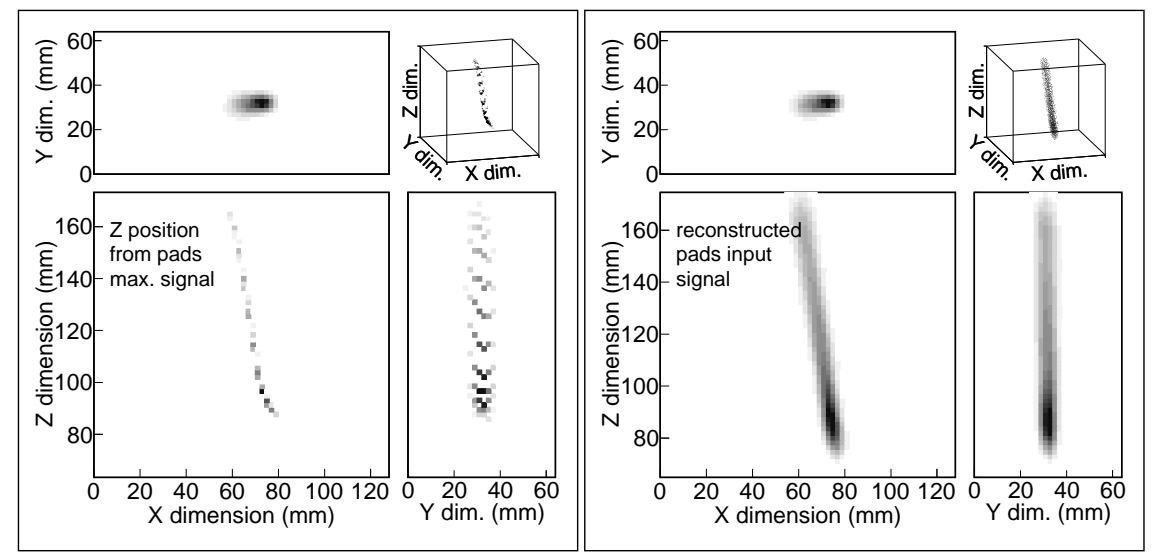

Figure 16: Example of close to vertical track illustrating the comparison of the 3D signal built from $2 \mathrm{D}$ amplitude $A\left(i_{x}, i_{y}\right)$ and time $T\left(i_{x}, i_{y}\right)$ analysis (left) and from the input signal $Z$ (or $T$ ) distribution reconstructed according to section 5.1 (right). In both cases, the $2 \mathrm{D}$ projections of signal amplitude are shown: $X$ and $Y$ are the collection pad plane dimensions and $Z$ is the signal drift dimension $\left(z=t \cdot v_{\text {drift }}\right)$. The $3 \mathrm{D}$ representation of the data is also plotted (upper-right corners).
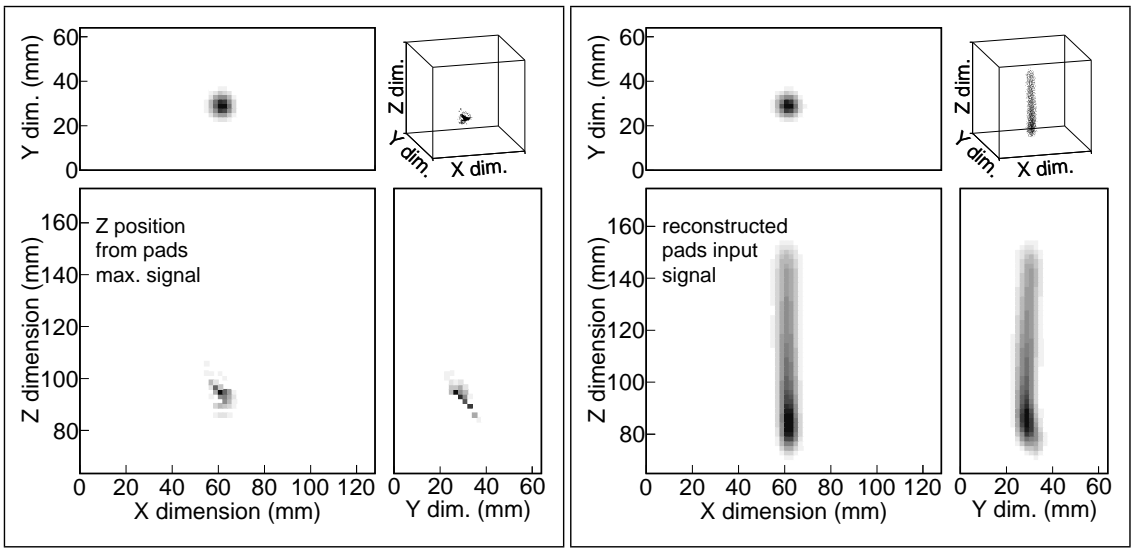

Figure 17: Same as figure 16 for an almost vertical track.

Concerning the particle trajectory reconstruction, the single timing informa${ }_{478}$ tion for each channel (time of maximum signal amplitude or CFD algorithm, 479 see 3) does not allow for an effective track reconstruction, as illustrated in figures 16 (example of a track with a small angle with respect to vertical axis) and 


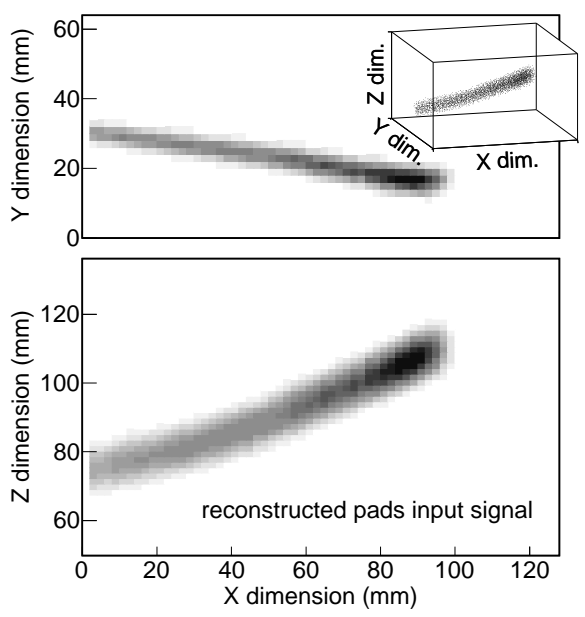

Figure 18: Example of signal reconstruction for the signal of an alpha particle from the source located on the side of the active volume. The upper plot is the signal projection on the $(X, Y)$ pad plane and the lower plot is the projection on the $(X, Z)$, the $Z$ axis being the drift direction (for which the input signal is reconstructed for each pad).

48117 (example of an almost vertical track). In both cases, the standard analysis 482 from $2 \mathrm{D}$ amplitude and time of pad signals is compared to the full 3D signal 483 reconstruction.

\subsection{Input signal reconstruction}

The full 3D input signal reconstruction is performed according to the channel signal processing technique proposed in ref. [11] (section 4).

The input charge distribution is reconstructed by deconvolution of the mea${ }_{488}$ sured (output) signal from the response function of the channel electronics es489 timated with the empirical method proposed in ref. [11]. The deconvolution 490 is performed in Fourier space using a fast Fourier transform (FFT) algorithm. 491 With $\left(i_{X}, j_{Y}\right)$ the pad index along $(X, Y)$ dimensions, and $f$ the frequency co492 ordinate for the Fourier transform of the sampling $T$ (or $Z$ ) dimension (the tilde 493 notation indicates Fourier transform), the reconstructed signal $S_{r e c}$ for a pad 494 is:

$$
\tilde{S}_{\text {rec }}\left[i_{X}, j_{Y}\right](f)=\frac{\tilde{S}_{\text {out }}\left[i_{X}, j_{Y}\right](f)}{\tilde{H}_{R F}\left[i_{X}, j_{Y}\right](f)} \cdot \tilde{\Phi}(f)
$$

where $S_{\text {out }}$ is the measured (output) signal, $H_{R F}$ is the electronics channel response function and $\Phi$ is a low-pass filter needed because of a small remaining noise in the response function. 
The output signal $S_{\text {out }}$ here is the sampled data after corrections mentioned in section 2.1. The channel-to-channel gain matching procedure (section 2.2) needs to be defined in the same conditions: the input signal reconstruction procedure is also applied on data taken with a pulse generator signal on the micromegas mesh before the gain matching calibration.

In addition to the right parts of figures 16 and 17 showing the reconstructed signal for tracks with the source on the top of the drift volume, figure 18 illustrates the case of the source located on the side.

\subsection{Track fit of reconstructed signal}

The reconstructed signal can be fitted with a $3 \mathrm{D}$ charge distribution function that is a generalization of the 2D function used for the $X Y$ signal distribution.

The signal at point $\vec{P}(x, y, z)$ in the active volume is the integral of the contributions from energy loss of all points $\vec{P}_{t r}(\varepsilon)$ along the track ( $\varepsilon$ is the track curve coordinate as defined in section 3.1 ):

$$
S_{3 D}(\vec{P})=\int_{\varepsilon=0}^{1} f_{3 D}(\vec{P}, \varepsilon) \cdot d \varepsilon
$$

with $f_{3 D}(\vec{P}, \varepsilon)$ the contribution of the charge deposited at track point $\vec{P}_{t r}(\varepsilon)$ to the active volume point $\vec{P}$, that can be expressed from the energy loss model along the track $f_{E}(\varepsilon)$ and the dispersion due to the drift of primary electrons:

$$
f_{3 D}(\vec{P}, \varepsilon)=f_{E}(\varepsilon) \cdot D_{X Y}(\vec{P}, \varepsilon) \cdot D_{Z}(\vec{P}, \varepsilon)
$$

The signal dispersion in $(X, Y)$ plane $D_{X Y}$ is the same as for the $2 \mathrm{D}$ analysis (eq. 4). For the dispersion along the drift axis $Z$, a Gauss function can also be used:

$$
D_{Z}^{(G)}(\vec{P}, \varepsilon)=\frac{1}{\sqrt{2 \pi} \cdot \sigma_{Z}(\varepsilon)} \cdot e^{-\frac{\left(z-z_{t r}(\varepsilon)\right)^{2}}{2 \cdot \sigma_{Z}(\varepsilon)^{2}}}
$$

with $\sigma_{Z}(\varepsilon)=\sigma_{Z}^{0}+\sigma_{Z}^{1} \cdot \sqrt{z_{t r}(\varepsilon)}$.

Due to the finite size of digitized signals, the FFT deconvolution and filtering induces a small distortion of the reconstructed signal distribution (see figure 19). It can be taken into account by replacing the $Z$ dispersion with the convolution of a Gauss function with a cardinal sine function, with an additional scaling parameter $\nu$ (when $\nu \rightarrow \infty$ the function becomes a Gaussian):

$$
D_{Z}^{(G S C)}(z, \varepsilon)=K(\varepsilon) \cdot e^{-\frac{\left(z-z_{t r}(\varepsilon)\right)^{2}}{2 \cdot \sigma_{Z}(\varepsilon)^{2}}} \cdot \operatorname{sinc}\left(\frac{\pi \cdot\left(z-z_{t r}(\varepsilon)\right)}{\nu \cdot \sigma_{Z}}\right)
$$

${ }^{524}$ with the factor $K(\varepsilon)$ defined so that the $D_{Z}$ dispersion function is normalized ${ }_{25}$ with respect to the integration over the $z$ variable: 


$$
K(\varepsilon)=\frac{1}{\nu \cdot \sigma_{Z}(\varepsilon) \cdot \operatorname{erf}\left(\frac{\pi}{\sqrt{2} \cdot \nu}\right)}
$$

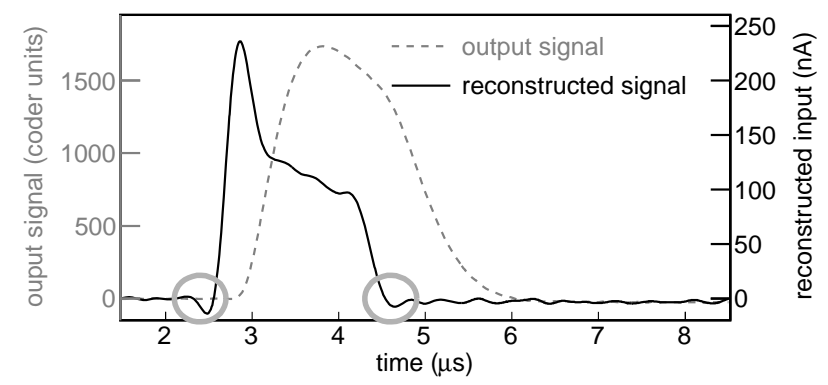

Figure 19: Example of input signal reconstruction for a single pad measured signal for a vertical track (taken from same event than fig. 17). The gray circles indicate the sidelobes resulting from the processing of finite size signals (FFT filtering). For the signal reconstruction, the sampling time window must be large enough to cover the full drift height, plus a time interval for the baseline amplitude offset analysis before the effective signal pulse (see section 2.1) and the baseline restoration after the signal.

The $Z$ dependence of the signal dispersion along the $Z$ axis is relatively small compared to the one for $(X, Y)$ dimensions: the smoothing effect of the filtering (in eq. 6) after deconvolution, that is constant with $z$, is dominating the observed width more than is the intrinsic signal dispersion. In addition, when using the $D_{Z}^{(G S C)}$ function, the observed width does not directly correspond to $\sigma_{Z}$ any more.

\subsection{Reconstructed signal analysis}

For the analysis of tracks from the reconstructed signals, the signal distribution (eq. 6) is fitted with the function from eq. 7. Since the 2D fit (amplitude and time, section 3) of vertical tracks is not possible, we compare the results for the $2 \mathrm{D}$ and $3 \mathrm{D}$ analysis for a measurement with source located on the side of the detector.

Table 1 summarizes the results comparison. For both measurement configurations (source on the side or on the top of the active volume), the analysis of the reconstructed signal gives results at least as good as the analysis of the output signal. For the measurement with the source on top, the results are somehow degraded. Nevertheless, in that case, the track fit was possible only with reconstructed signal. With the source located on the detector side (horizontal tracks), the output and the reconstructed signals analysis can be compared and 


\begin{tabular}{|c|c|c|c|}
\hline $\begin{array}{l}\text { source } \\
\text { position }\end{array}$ & $\begin{array}{l}\text { analysed } \\
\text { signal }\end{array}$ & $\begin{array}{l}\text { energy resolution } \\
(\mathrm{keV})^{(1)}\end{array}$ & $\begin{array}{l}\text { length resolution } \\
(\mathrm{mm})\end{array}$ \\
\hline \multirow[t]{2}{*}{$\begin{array}{l}\text { side } \\
\text { (fig. 1a) }\end{array}$} & output & $\begin{array}{l}79.8 \pm 5.3^{(2)} \\
79.4 \pm 5.2^{(3)}\end{array}$ & $3.43 \pm 0.13$ \\
\hline & reconstructed & $74.4 \pm 5.0$ & $\begin{array}{l}3.22 \pm 0.12^{(4)} \\
3.17 \pm 0.08^{(5)}\end{array}$ \\
\hline \multirow{2}{*}{$\begin{array}{l}\text { top } \\
\text { (fig. 1b) }\end{array}$} & output & $109.5 \pm 8.1^{(3,6)}$ & no tracks fitting \\
\hline & reconstructed & $107.8 \pm 7.5$ & $\begin{array}{l}4.45 \pm 0.16^{(4)} \\
4.44 \pm 0.18\end{array}$ \\
\hline
\end{tabular}

Table 1: Comparison of the resolution (energy and fitted track length) from the reconstructed signal (full $3 \mathrm{D}$ ) and from the from the output signal $(2 \mathrm{D}$ amplitude and time) analysis.

(1) for energy resolution, a maximum angle $10^{\circ}$ is considered (see section 3.3)

(2) from pads signal maximum amplitude

(3) from pads integral signal over threshold

(4) with $Z$ signal dispersion $D_{Z}^{(G)}$ from eq. 9

(5) with $Z$ signal dispersion $D_{Z}^{(G S C)}$ from eq. 10

(6) track angles from $3 D$ track fit with reconstructed signal

we did not observe any disadvantage of the reconstruction, such as biases or non-linearities.

For the measurement with the source on the side, figure 20 illustrates the better quality of the Bragg peak fit in the case of the analysis of the reconstructed signal, compared to the output signal.

\section{Conclusion}

We have investigated the evolution of the performance of the ACTAR TPC device when exploring the main parameters for the configuration of the GET electronics and the detector settings. After the raw data corrections, the best results are obtained with a larger peaking time (mainly due to a better signalto-noise ratio). The readout depth (sampling size) can be reduced (from 512 to 256) with no significant degradation thus allowing a factor 2 reduction of the data volume and the event acquisition dead-time. The full readout mode is preferable to partial readout, but it may not be used in experiments because of a too large data rate. Nevertheless, the trigger threshold may be set low enough for an acceptable analysis precision. We noticed significantly better results with a larger drift velocity, keeping in mind that it may be related to a small gas pollution with air.

We observed a discrepancy between the drift velocity estimated from the data and from the GARFIELD program. Since we could not conclude on the reasons of the disagreement, we would like to emphasis the importance of con- 


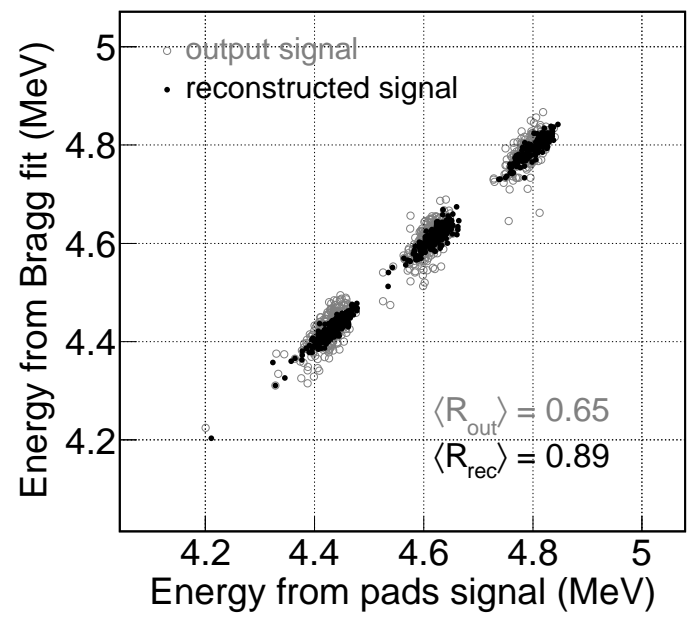

Figure 20: Comparison of the correlation between the energy measured from the pads signal amplitude (abcissa) and from the Bragg peak parameters of the fit (ordinate), in the measurement with the source on the side of the TPC. The correlation coefficients result from the average for the 3 peaks. The correlation is better for the fit of tracks with the reconstructed signal (black dots) than with the output signal (gray circles), indicating a better fit of the Bragg peak.

sidering an experimental determination of this velocity for the effective run conditions of any on-site measurement.

We have demonstrated the importance of a full reconstruction of the input charge collection, by deconvolution of the registered (output) data samples from the channel response function in the case of tracks close to the drift direction. This may be of particular importance for decay experiments with stopped ions (the decay particles are emitted in $4 \pi$ ) or in experiments using active targets with incident beam parallel to the drift axis.

\section{Acknowledgment}

The research leading to these results have received funding from the European Research Council under the European Union's Seventh Framework Program (FP7/2007-2013)/ERC grant agreement no 335593 and from the Conseil Régional d'Aquitaine (grant no 2014-1R60402 - 00003319).

\section{References}

[1] T. Roger and et al., Nucl. Instrum. Methods Phys. Res. A 895, 126 (2017). 
[2] J. Giovinazzo and et al., Nucl. Instrum. Methods Phys. Res. A892, 114 (2018).

[3] B. Mauss and et al., Nucl. Instrum. Methods Phys. Res. A 940, 498 (2019).

[4] E. Pollacco and et al., Nucl. Instrum. Methods Phys. Res. A887, 81 (2018).

[5] D. Suzuki et al., Nucl. Instrum. Methods Phys. Res. A691, 39 (2013).

[6] D. Bazin et al., Active Targets and TPC for Nucl. Phys. Exp. Workshop, MSU, $\quad$ https://indico.fnal.gov/contributionDisplay. py? contribId=38EsessionId=1ÉconfId =8976, (2015).

[7] T. Isobe et al., Nucl. Instrum. Methods Phys. Res. A899, 43 (2018).

[8] I. Giomataris and et al., Nucl. Instrum. Methods Phys. Res. A560, 405 (2006).

[9] J. Alison et al., Nucl. Instrum. Methods Phys. Res. A835, 186 (2016).

[10] Garfield, http://garfield.web.cern.ch/garfield, simulation of gaseous detectors .

[11] J. Giovinazzo and et al., Nucl. Instrum. Methods Phys. Res. A840, 15 (2016).

[12] B. Mauss et al., EPJ Web of Conferences 174, 01010 (2015).

[13] P. Baron and E. Delagnes, AGET Data Sheet, project-get.cea.fr/Public/100_workgroups/300_wp2/100_aget/aget_data_sheet_v31156/view, (2015). 